\title{
Can Collagenase Be Used in the Treatment of Adhesive Capsulitis?
}

\author{
Nazım Karahan ${ }^{a}$ Güzelali Ozdemir $^{b}$ Duygu Kolukısa ${ }^{c}$ Serda Duman $^{d}$ \\ Fatih Arslanoğlue Mehmet Çetin ${ }^{f}$ \\ aDepartment of Orthopaedics and Traumatology, Corlu State Hospital, Tekirdağ, Turkey; ${ }^{b}$ Department of \\ Orthopaedics and Traumatology, Ankara Numune Education and Research Hospital, Ankara, Turkey; ' Department \\ of Pathology, Mersin City Hospital, Mersin, Turkey; ${ }^{\mathrm{d} D e p a r t m e n t ~ o f ~ O r t h o p a e d i c s ~ a n d ~ T r a u m a t o l o g y, ~ D i y a r b a k i r ~}$

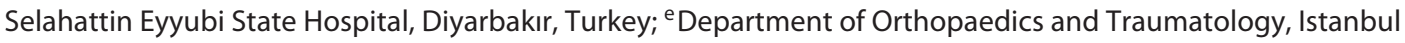 \\ Medipol Universitesi, Istanbul, Turkey; ${ }^{\mathrm{f}}$ Department of Orthopaedics and Traumatology, Fatih Sultan Mehmet \\ Education and Research Hospital, Istanbul, Turkey
}

\section{Highlights of the Study}

- Subacromial administration of collagenase was more effective than steroid and saline in improving shoulder abduction angle and in terms of histological/histochemical examination.

- Collagenase administered subacromially did not pass into the glenohumeral space, and therefore did not cause chondral damage.

- There were no significant differences in abduction angles and histological/histochemical examination between steroid and saline groups.

\section{Keywords}

Adhesive capsulitis · Collagenase · Experimental study · Rat · Steroid

\footnotetext{
Abstract

Background: The objective of this study was to evaluate the efficacy of subacromial injections of collagenase and corticosteroid in rats with experimentally induced adhesive capsulitis. Method: Thirty adult Wistar albino male rats were distributed into 3 groups of 10 rats each after stabilization of their shoulders for 3 weeks: the first group received a single dose of $0.002 \mathrm{mg}(0.25 \mathrm{~mL})$ subacromial collagenase; the second group received a single dose of $1.60 \mathrm{mg}(0.25$ $\mathrm{mL}$ ) subacromial steroid, and the third group received a single dose of $0.25 \mathrm{~mL}$ subacromial saline solution. One week later, we investigated shoulder range of motions, collagen
}

\begin{tabular}{ll}
\hline KARGER & ( 2019 The Author(s) Karger \\
& Published by S. Karger AG, Basel Open ciccess \\
karger@karger.com & This is an Open Access article licensed under the Creative Commons \\
www.karger.com/mpp & $\begin{array}{l}\text { Attribution-NonCommercial-4.0 International License (CC BY-NC) } \\
\text { (http://www.karger.com/Services/OpenAccessLicense), applicable to } \\
\text { the online version of the article only. Usage and distribution for com- } \\
\text { mercial purposes requires written permission. }\end{array}$
\end{tabular}

content of the shoulder, and joint cartilage structure. $\boldsymbol{R} \boldsymbol{e}$ sults: There was no statistically significant difference in the cartilage damage between the groups $(p>0.05)$. Fibrosis measurements were significantly lower in the collagenase group than in the steroid and saline groups. There was no significant difference in fibrosis between the steroid and saline groups $(p>0.05)$. Abduction measurements were significantly higher in the collagenase group than in the steroid and saline groups ( $p<0.001)$. No significant difference in the abduction measurements was observed between the saline and steroid groups ( $p>0.05)$. Conclusion: We observed that subacromial injections of collagenase Clostridium histolyticum effectively treated adhesive capsulitis. The results suggest that this treatment could be considered for use in patients with an intact rotator cuff.

C 2019 The Author(s)

Published by S. Karger AG, Basel

Nazım Karahan

Department of Orthopaedics and Traumatology, Corlu State Hospital

Zafer Mahallesi, Bülent Ecevit Blv. No: 33

TR-34752 Tekirdağ (Turkey)

E-Mail usta_84@hotmail.com 


\section{Introduction}

Adhesive capsulitis of the shoulder is characterized by a gradual onset of shoulder pain and limited passive and active range of motions (ROM) with respect to external rotation and forward flexion which affects $2-5 \%$ of the general population [1]. Its incidence increases in systemic diseases, especially in insulin-dependent diabetes mellitus [2].

Adhesive capsulitis is a disease characterized by fibroblast proliferation, thickening of both the coracohumeral ligament (CHL) and capsule at the rotator cuff interval, and complete obliteration of the fat triangle under the coracoid process and axillary recess [3]. The microscopic examination of tissue from sufferers of adhesive capsulitis reveals fibroblasts mixed with type I and type III collagen. The fibroblasts change into smooth muscle phenotype (myofibroblasts), which appears to cause the capsular contraction [4], but the etiology and pathophysiology of adhesive capsulitis have not been fully elucidated [5].

Fibroplasic changes, increased local collagen, and myofibroblasts are observed in Dupuytren's disease as well as in Peyronie's disease $[6,7]$. Adhesive capsulitis pathoanatomy was observed to be quite similar $[8,9]$.

Collagenase Clostridium histolyticum $(\mathrm{CCH})$ consists of two synergistic collagenases (CCH-I and CCH-II). It is an effective enzymatic injection used in the treatment of Dupuytren's disease and Peyronie's disease [10].

The objective of this study was to investigate the activity of intralesional $\mathrm{CCH}$ in rats with experimental adhesive capsulitis because it has a similar pathoanatomy.

\section{Materials and Methods}

Thirty adult male 7-month-old Wistar albino rats with an average weight of 220-275 g were included in the study. The rats were obtained from the Experimental Animal Breeding and Research Laboratory of Diele University. The animals were kept at $20-24{ }^{\circ} \mathrm{C}$, $50-55 \%$ relative humidity, under a 12 -h light/12-h dark cycle in a noiseless environment. They were fed with standard laboratory food without liquid or food restriction.

\section{Frozen Shoulder Modeling}

The rats were anesthetized with intramuscular xylazine hydrochloride (5 mg/kg, Rompun ${ }^{\circledR}$; Bayer, Germany) and ketamine hydrochloride $\left(50 \mathrm{mg} / \mathrm{kg}\right.$, Ketalar ${ }^{\mathrm{TM}}$; Pfizer, USA) at time zero. The depth of the anesthesia was assessed by monitoring the corneal reflex and responses to painful stimulation of the foot. The shoulder, including the whole left extremity, was bandaged with compression to the body, the shoulder was in adduction and internal rotation, and the elbow was placed in flexion and pronation so that it would not become loose and also not prevent respiration and
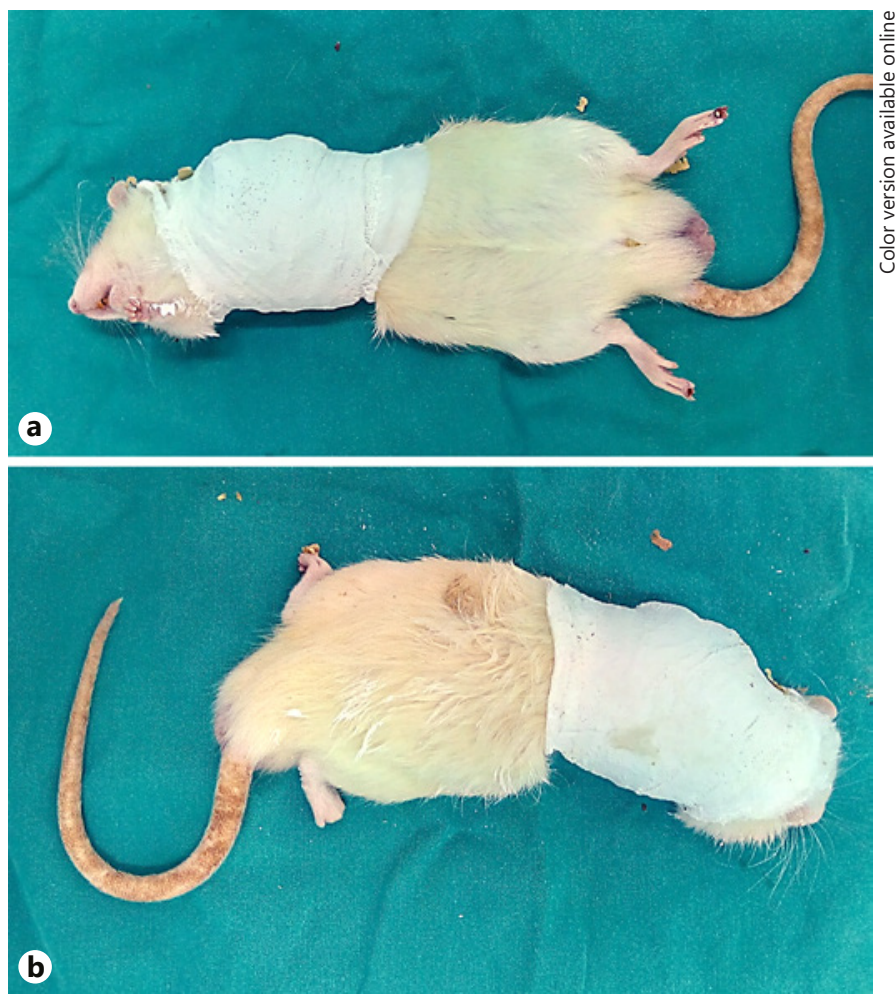

Fig. 1. Immobilization of the shoulder in a rat model. Immobilization was achieved by molding plaster around the left shoulder at $90^{\circ}$ of internal rotation, after anesthesia.

circulation. No food and fluid restrictions were applied. The animals were checked daily to monitor any loosening or rupture of the bandage [11] (Fig. 1).

The animals were allowed to move freely for 3 weeks, and then the shoulder bandages were removed after they were anesthetized with intramuscular xylazine hydrochloride $(5 \mathrm{mg} / \mathrm{kg}$, Rompun; Bayer) and ketamine hydrochloride (50 mg/kg, Ketalar; Pfizer). The animals were randomized into 3 groups of 10 rats each. A randomization table assigned the digits to the collagenase, the steroid, or the saline groups. The first group (collagenase group) received subacromial $0.002 \mathrm{mg}(0.25 \mathrm{~mL}) \mathrm{CCH}$ (Xiaflex ${ }^{\complement}$, USA). The second group (steroid group) received $1.60 \mathrm{mg}(0.25 \mathrm{~mL})$ betamethasone dipropionate (Dipraspan ${ }^{\circledR}$; Schering Plough, USA). The third group (saline group) received $0.25 \mathrm{~mL}$ saline solution. Saline (isotonic sodium chloride solution) was also administered subacromially. Surgery was performed using a lateral approach to ensure that the injected material was in the subacromial area. After skin incision, a longitudinal incision was made on the deltoid muscle to expose the rotator cuff tendons at the left shoulder joint. The deltoid muscle and skin were closed by using 5-0 nylon sutures in 3 groups. The dosage was calculated from the amount of $\mathrm{CCH}$ per kg human body weight administered to people with Dupuytren's disease.

The animals were again allowed to move freely for 7 days, and then the abduction angles were measured with the rats under anesthesia and then sacrificed by decapitation. The midline of the humeral diaphysis was removed from the scapular periscapular 

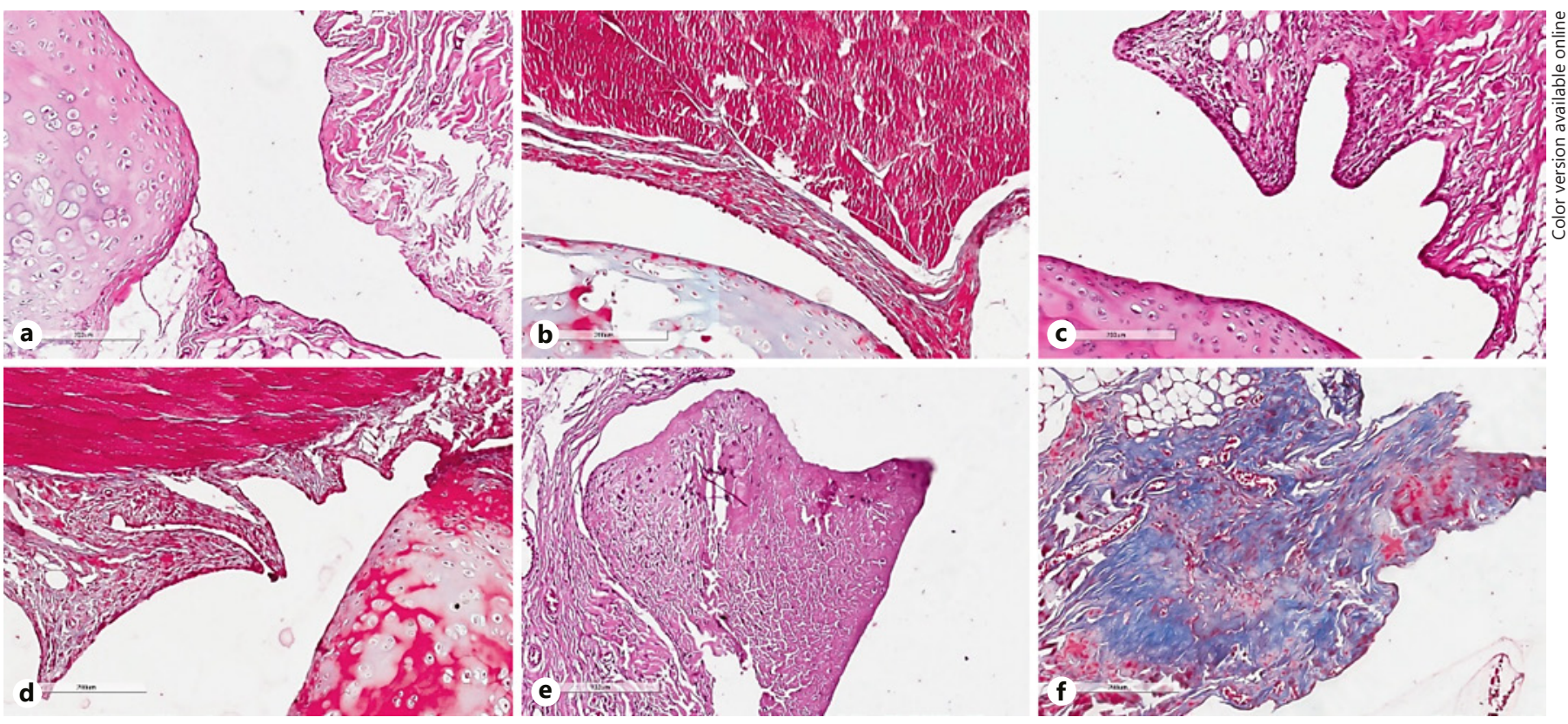

Fig. 2. Different fibrosis grades in the collagenase, steroid, and control experimental groups. a H\&E collagenase. b Masson trichrome collagenase. c H\&E steroid. d Masson trichrome steroid. e H\&E control. f Masson trichrome control. a, b A sample from the subjects in the collagenase group without fibrosis (H\&E vs. Masson 295 trichrome, $\times 200$ ). c, d Mild severe fibrosis observed in a subject in the steroid group (H\&E vs. Masson 297 trichrome, $\times 200$ ). e, f Medium-severity fibrosis observed in the control group (H\&E vs. Masson trichrome, $\times 200$ ).

muscles after half osteotomy of the clavicle, and the shoulder joint was completely removed [12]. The excised materials were prepared for histological and histochemical examination.

\section{Measurement of the Abduction Angle}

After removal of the shoulder bandage, the angle between the humeral shaft and spina scapula under $10 \mathrm{~g}$ of torque $(3.92 \times$ $10-3.78 \mathrm{~N} \times \mathrm{m}$ ) was measured to evaluate the maximum passive abduction [12]. To assess the abduction angles, the angle formed from the intersection of a line on the scapular spine and a line originating from the center of the humeral head to that of the humeral condyle was measured by using a goniometer. The shoulder ROMs was measured 3 times, and the average value of these measurements was used for further analysis.

\section{Histological Examination}

The specimens were stored in a pathology container with $10 \%$ formaldehyde. The materials were numbered by using a randomization method. The specimens were fixed for 1 week and then underwent a decalcification process (DDK ${ }^{\mathrm{TM}}$ ) for 5 days. Sampling with a thickness of $4 \mathrm{~mm}$ was performed to evaluate the joint space and synovial membranes. The samples were placed in an automatic tissue-processing device (MTM1 ${ }^{\mathrm{TM}}$; SLEE, Germany) for $13 \mathrm{~h}$. Tissues were embedded into the paraffin, and then sections $(4-\mu \mathrm{m}$ thickness) were prepared. Samples were stained with hematoxylin and eosin, ScyTekTRM-2 (Trichrome Stain Kit, Modified Masson's), and SOH250 safranin-O solution kits. The samples were examined by a pathologist blinded to study. Sections were examined under a light microscope (Nikon Eclipse Ci; Nikon, Japan).
Fibrosis was assessed in the capsule at the rotator interval in the receiving material. Fibrosis was categorized into 3 groups according to the staining level with modified Masson's stain as slight, moderate, and intensive [13] (Fig. 2).

The changes in the structure of the head of humerus cartilage were evaluated according to the modified Mankin's scale. In the modified Mankin's scoring system, the properties of the joint cartilage structure, number of cells, staining pattern with safranin$\mathrm{O}$, and tidemark zone were evaluated and scored in 4 different categories. The scoring system has a scale between 0 and 14 [14] (Table 1).

\section{Statistical Analysis}

The SPSS v25.0 software package (IBM Corporation, Armonk, NY, USA) was used for the statistical analysis. The normal distribution of the data was evaluated by performing the Shapiro-Wilk test. Variance homogeneity was assessed by the Levene test. The one-way ANOVA (robust test: Brown-Forsythe) test and the Fisher's least significant difference test were used for parametric methods to compare the collagenase, steroid, and saline groups with each other according to the shoulder ROM quantitative data. The Kruskal-Wallis $\mathrm{H}$ test was used to analyze the Monte Carlo simulation technique results for the nonparametric tests of the total Mankin's score. The Fisher-Freeman-Holton test was performed by using the Monte Carlo simulation technique for comparison of the collagenase, steroid, and saline groups according to the variables of fibrosis, safranin-O staining, structure, cellularity, and tidemark variables, and column ratios were compared with each other and expressed according to Benjamini-Hochberg-corrected 
Table 1. Mankin's score

\begin{tabular}{ll}
\hline Structure & Normal (0) \\
& Surface irregularities (1) \\
& Pannus and surface irregularities (2) \\
& Clefts to transitional zone (3) \\
& Cleft to radial zone (4) \\
& Cleft to calcified zone (5) \\
& Complete disorganization (6) \\
& Normal (0) \\
& Diffuse hypercellularity (1) \\
& Cloning (2) \\
& Hypocellularity (3) \\
\hline Safranin-O staining & Normal (0) \\
& Slight reduction (1) \\
& Moderate reduction (2) \\
& Severe reduction (3) \\
& No dye noted (4) \\
\hline Tidemark integrity & Intact (0) \\
& Crossed by blood vessels (1) \\
\hline Maximal score & 14 (normal =0) \\
\hline
\end{tabular}

$p$ values. Quantitative variables are expressed as the mean $\pm \mathrm{SD}$ (standard deviation) - minimum/maximum and median (minimum/maximum), and categorical variables are shown as $n(\%)$ in the tables. Variables were examined at the $95 \%$ confidence level, and $p<0.05$ was accepted as indicative of statistical significance.

\section{Results}

\section{Gross Observation}

No infection, severe weight loss, or mortality was observed in the experimental animals after the surgical intervention. The rotator cuff was intact in all groups. No local skin problems or ulcers were observed in any group.

Serial histological evaluations were performed in all groups. The most noticeable changes observed were muscle atrophy, the disappearance of the synovial fold, and sub-synovial fat tissue. In all groups, infiltration of lymphoplasmacytoid cells was observed in the synovium.

\section{Abduction Arm Angle}

There was a statistically significant difference in shoulder abduction between the groups $(p<0.05)$. The double comparison, which was performed to determine the difference-making group, showed that abduction measurements were significantly higher in the collagenase group than in the steroid and saline groups $(p<0.001)$. No significant difference in abduction arm angle was observed between the saline and steroid groups $(p>0.05)$ (Table 2).

\section{Mankin's Score}

There was no significant difference in the total Mankin's scores between the groups $(p>0.05)$. There were no significant differences in the measurements of tidemark continuity, safranin-O staining, cellularity, and cartilage structure between the groups $(p>0.05)$.

\section{Histochemical Staining}

There was a statistically significant difference in fibrosis between the groups $(p=0.012)$. A double comparison, which was performed to determine the difference-making group, showed that fibrosis measurements were significantly lower in the collagenase group than in the steroid and saline groups. No significant difference in fibrosis was observed between the saline and steroid groups. All the samples had some degree of fibrosis after shoulder bandage and injections.

\section{Discussion}

A search of the literature showed that there are no reports on the effect of $\mathrm{CCH}$ on adhesive capsulitis in experimental animals. In our study, we observed that subacromial injection of $\mathrm{CCH}$ was more effective in the adhesive capsulitis model than in the steroid group and saline (control) group $(p<0.001)$. Our study demonstrated that single doses of subacromial collagenase, steroid, and saline injections did not cause cartilage damage $(p>0.05)$.

There is only one clinical study on the treatment of $\mathrm{CCH}$ adhesive capsulitis in the literature. In this study, extra-articular CCH injections were observed to be effective and tolerable in the treatment of adhesive capsulitis. The small study number and the variability of the diagnosis of adhesive capsulitis by clinical examination are limitations of this study [15].

Investigation of the rotator interval capsule and $\mathrm{CHL}$ obtained from adhesive capsulitis patients revealed active fibroblastic proliferation accompanied by some transformation to myofibroblasts, with inflammation and synovial involvement, which was very similar to the characteristics in Dupuytren's disease $[7,16]$. In our study, infiltration of lymphoplasmacytoid cells was observed in the synovium in all groups.

$\mathrm{CCH}$ is currently used in the treatment of Dupuytren's disease, Peyronie's disease, and necrotic wounds 
Table 2. Mankin's score, fibrosis, and shoulder range of motion according to the groups

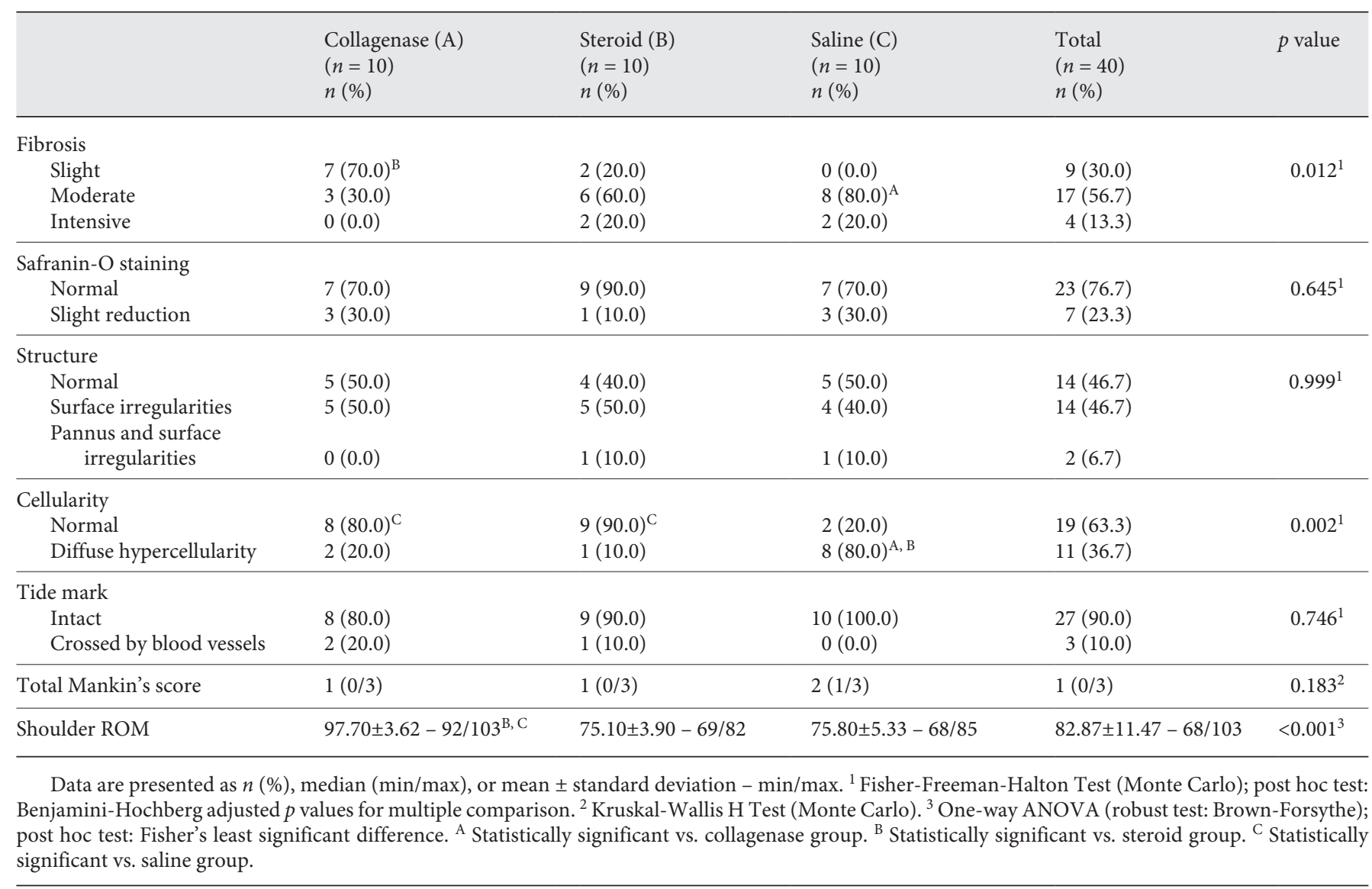

as an inducer of enzymatic debridement. $\mathrm{CCH}$ is the first agent approved in the US and EU for nonsurgical treatment of Dupuytren's contracture [17]. Clinical studies and postmarketing trials have confirmed that $\mathrm{CCH}$ is an effective and safe treatment for Dupuytren's disease $[18,19]$.

Intralesional injection of $\mathrm{CCH}$ for the treatment of Peyronie's disease is a recent innovation that seeks to bridge the gap in efficacy between minimally invasive therapies and established invasive surgical therapies. $\mathrm{CCH}$ has previously been used to great effect in pathogenically similar disease processes such as Dupuytren's contracture and appears to be well tolerated [20].

$\mathrm{CCH}$ hydrolyzes collagen in its entirety at the level of the triple helix. However, not all types of collagen are degraded by $\mathrm{CCH}$ [21]. $\mathrm{CCH}$ degrades type 1, type 2, type 3 collagens in vivo, and collagen type 4 only in vitro [22]. $\mathrm{CCH}$ causes a decrease in extracellular matrix and cytokines and stimulates growth factors; it destroys collagen fibers and also suppresses their adhesion sites [23]. CCH decreases $\alpha$-smooth muscle actin, transforming growth factor- $\beta$, fibronectin, desmin, and fibroblast activity [24]. All these activities cause a cumulative decrease in the synthesis of abnormal type 1 and type 3 collagens and destruction of pathological collagen plates. Only high doses and repeated administrations have an effect on type 4 collagen. This is important because type 4 collagen is a connective tissue component surrounding the vessels [24].

A total of 890 patients with Dupuytren's disease were treated with $\mathrm{CCH} ; 13 \%$ had skin problems, $9.5 \%$ had peripheral edema, 9.7\% had contusions, and $0.05 \%$ had tendon ruptures [25]. Anaphylactic reaction due to the $\mathrm{CCH}$ has been reported only as a case report in the literature [26]. The most common adverse immunological effects were lymphadenopathy and axillar pain [27]. In our study, no complications, such as rotator cuff damage or skin problems, were observed in the groups during the gross pathological examinations.

Most of the studies have indicated that steroids are effective agents in the improvement of pain and function, 
especially in the early period, with a low rate of side effects, but current comparative clinical studies have not been able to demonstrate any difference between corticosteroid and saline in long-term functional improvement $[28,29]$. Similarly, we did not detect any differences in abduction angles and histological/histochemical examination results between the steroid and saline groups.

Frozen shoulder modeling in mice, rats, and dogs is available in many formats. The rat shoulder contracture model, which is carried out with immobilization, has the advantage of similarity of the rat anatomy to the human anatomy [12]. Several methods have been used to achieve shoulder contracture. Molding plaster or extra-articular fixation using a plastic plate or suture material has been used $[11,30]$. In the current study, we chose a rat shoulder contracture model using molding plaster because it has been shown to be a simple and effective secondary adhesive capsulitis model with high reproducibility [12].

In our study, subacromial injection was preferred because of the primary pathology of the CHL and rotator interval in adhesive capsulitis [31], and it has been observed that intra-articular applications of collagenases cause chondral damage even at low doses [32]. Intra-articular collagenase administration is not preferred as it causes chondral damage and does not affect the $\mathrm{CCH}$ ligament and periarticular structure [15]. It can be suggested that subacromial injection of collagenase may reach the glenohumeral joint because of the collagen content of the rotator cuff and the rotator interval. However, the absence of any difference in the Mankin's score revealed that the single dose of subacromial injected $\mathrm{CCH}$ had no degenerative intraarticular effect. Similarly, Badalamente and Wang [15] did not observe rotator cuff and cartilage damage in their clinical study.

This study has several limitations. First, CHL, which has an important role in the pathology of adhesive capsu- litis, was not evaluated. There is no model for evaluating fibrosis in CHL in the literature. Similarly, $\mathrm{CCH}$ has not been analyzed on the muscles. Second, subacromial CCH injection does not affect the inferior capsule, which is important for the treatment of adhesive capsulitis. Third, rat shoulder function might be different from the human shoulder; rats are quadrupeds, which means that the forelimb is load-bearing [30]. Our experimental animal model causes secondary adhesive capsulitis rather than primary adhesive capsulitis. Fourth, only the abduction angle was evaluated in our study. Adhesive capsulitis is a progressive process in which the limitation of active and passive ROMs is limited in all directions, and restriction of ROM usually starts with external rotation in human shoulders that had been frozen, followed by abduction [1]. As only the abduction angle was measured in animal modeling, the external rotation measurements were not evaluated in our study $[11,12]$.

\section{Conclusion}

This study demonstrates that subacromial injection of $\mathrm{CCH}$ was an effective treatment for adhesive capsulitis; we suggest that it could be especially effective in patients with an intact rotator cuff.

\section{Statement of Ethics}

This experimental study was approved by the Ethics Committee for Animal Experiments of Dicle University (2017, No.: 11).

\section{Disclosure Statement}

The authors declare no conflicts of interest.

\section{References}

1 Neviaser AS, Neviaser RJ. Adhesive capsulitis of the shoulder. J Am Acad Orthop Surg. 2011 Sep;19(9):536-42.

2 Sattar MA, Luqman WA. Periarthritis: another duration-related complication of diabetes mellitus. Diabetes Care. 1985 Sep-Oct;8(5): $507-10$.

3 Itoi E, Berglund LJ, Grabowski JJ, Naggar L, Morrey BF, An KN. Superior-inferior stability of the shoulder: role of the coracohumeral ligament and the rotator interval capsule. Mayo Clin Proc. 1998;73(6):508-15.

4 Rodeo SA, Hannafin JA, Tom J, Warren RF, Wickiewicz TL. Immunolocalization of cyto-

Collagenase in the Treatment of Adhesive Capsulitis kines and their receptors in adhesive capsulitis of the shoulder. J Orthop Res. 1997 May; 15(3):427-36

5 Bunker T. Time for a new name for frozen shoulder-contracture of the shoulder. Shoulder Elbow. 2009;1(1):4-9.

6 Jordan GH. The use of intralesional clostridial collagenase injection therapy for Peyronie's disease: a prospective, single-center, non-placebo-controlled study. J Sex Med. 2008 Jan; 5(1):180-7.

7 Bunker TD, Anthony PP. The pathology of frozen shoulder. A Dupuytren-like disease. J Bone Joint Surg Br. 1995 Sep;77(5):677-83.
8 Shah N, Lewis M. Shoulder adhesive capsulitis: systematic review of randomised trials using multiple corticosteroid injections. $\mathrm{Br} \mathrm{J}$ Gen Pract. 2007 Aug;57(541):662-7.

9 Smith SP, Devaraj VS, Bunker TD. The association between frozen shoulder and Dupuytren's disease. J Shoulder Elbow Surg. 2001 Mar-Apr;10(2):149-51.

10 Xiaflex $^{\circledR}$. Collagenase clostridium histolyticum. Auxilium Pharmaceuticals, Inc. Available at: http://www.endo.com/File\%20Library/Products/Prescribing\%20Information/ Xiaflex_prescribing_information.html. Accessed March 8, 2016. 
11 Liu YL, Ao YF, Cui GQ, Zhu JX. Changes of histology and capsular collagen in a rat shoulder immobilization model. Chin Med J (Engl). 2011 Dec;124(23):3939-44.

12 Kim DH, Lee KH, Lho YM, Ha E, Hwang I, Song KS, et al. Characterization of a frozen shoulder model using immobilization in rats. J Orthop Surg Res. 2016 Dec;11(1):160.

13 Cake MA, Smith MM, Young AA, Smith SM, Ghosh P, Read RA. Synovial pathology in an ovine model of osteoarthritis: effect of intraarticular hyaluronan (Hyalgan). Clin Exp Rheumatol. 2008 Jul-Aug;26(4):561-7.

14 Pester JK, Stumpfe S, Steinert S, Marintschev I, Aurich M, Hofmann GO. BMP-2 shows characteristic extracellular patterns in osteoarthritic cartilage: a preliminary report. GMS Interdiscip Plast Reconstr Surg DGPW. 2013 Aug;2:Doc09.

15 Badalamente MA, Wang ED. CORR ${ }^{\circledR}$ ORS Richard A. Brand Award: Clinical trials of a new treatment method for adhesive capsulitis. Clin Orthop Relat Res. 2016 Nov;474(11): 2327-36.

16 Bunker TD, Reilly J, Baird KS, Hamblen DL. Expression of growth factors, cytokines and matrix metalloproteinases in frozen shoulder. J Bone Joint Surg Br. 2000 Jul;82(5):768-73.

17 Warwick D, Arandes-Renú JM, Pajardi G, Witthaut J, Hurst LC. Collagenase Clostridium histolyticum: emerging practice patterns and treatment advances. J Plast Surg Hand Surg. 2016 Oct;50(5):251-61.

18 Witthaut J, Jones G, Skrepnik N, Kushner H, Houston A, Lindau TR. Efficacy and safety of collagenase clostridium histolyticum injection for Dupuytren contracture: short-term results from 2 open-label studies. J Hand Surg Am. 2013 Jan;38(1):2-11.
19 Hurst LC, Badalamente MA, Hentz VR, Hotchkiss RN, Kaplan FT, Meals RA, et al.; CORD I Study Group. Injectable collagenase clostridium histolyticum for Dupuytren's contracture. N Engl J Med. 2009 Sep;361(10): 968-79.

20 Peak TC, Mitchell GC, Yafi FA, Hellstrom WJ. Role of collagenase clostridium histolyticum in Peyronie's disease. Biologics. 2015;9: 107-16.

21 French MF, Mookhtiar KA, Van Wart HE. Limited proteolysis of type I collagen at hyperreactive sites by class I and II Clostridium histolyticum collagenases: complementary digestion patterns. Biochemistry. 1987 Feb; 26(3):681-7.

22 Toyoshima T, Matsushita O, Minami J, Nishi $\mathrm{N}$, Okabe A, Itano T. Collagen-binding domain of a Clostridium histolyticum collagenase exhibits a broad substrate spectrum both in vitro and in vivo. Connect Tissue Res. 2001; 42(4):281-90

23 Palmieri A, Imbimbo C, Longo N, Fusco F, Verze $\mathrm{P}$, Mangiapia F, et al. A first prospective, randomized, double-blind, placebo-controlled clinical trial evaluating extracorporeal shock wave therapy for the treatment of Peyronie's disease. Eur Urol. 2009 Aug;56(2): 363-9.

24 Levine LA, Schmid TM, Emeigh Hart SG, Tittelbach T, McLane MP. Collagenase clostridium histolyticum degrades type I and III collagen while sparing type IV collagen in vitro in Peyronie's plaque explants. Urological Association Annual Meeting; 2014; Orlando; abstract PD22-03.
25 Peimer CA, McGoldrick CA, Kaufman G. Nonsurgical treatment of Dupuytren contracture: 3-year safety results using Collagenase Clostridium histolyticum. J Hand Surg Am. 2013;38(10):e52.

26 Gaston RG, Larsen SE, Pess GM, Coleman S, Dean B, Cohen BM, et al. The Efficacy and Safety of Concurrent Collagenase Clostridium Histolyticum Injections for 2 Dupuytren Contractures in the Same Hand: A Prospective, Multicenter Study. J Hand Surg Am. 2015 Oct;40(10): 1963-71.

27 Sanjuan-Cerveró R, Carrera-Hueso FJ, Vazquez-Ferreiro P, Gomez-Herrero D. Adverse effects of collagenase in the treatment of dupuytren disease: A Systematic Review. BioDrugs. 2017 Apr;31(2):105-15.

28 Roh YH, Yi SR, Noh JH, Lee SY, Oh JH, Gong $\mathrm{HS}$, et al. Intra-articular corticosteroid injection in diabetic patients with adhesive capsulitis: a randomized controlled trial. Knee Surg Sports Traumatol Arthrosc. 2012 Oct;20(10): 1947-52.

29 Bal A, Eksioglu E, Gulec B, Aydog E, Gurcay E, Cakci A. Effectiveness of corticosteroid injection in adhesive capsulitis. Clin Rehabil. 2008 Jun;22(6):503-12.

30 Kanno A, Sano H, Itoi E. Development of a shoulder contracture model in rats. J Shoulder Elbow Surg. 2010 Jul;19(5):700-8.

31 Ryan V, Brown H, Minns Lowe CJ, Lewis JS. The pathophysiology associated with primary (idiopathic) frozen shoulder: A systematic review. BMC Musculoskelet Disord. 2016;15; 17(1):340.

32 Yeh TT, Wen ZH, Lee HS, Lee CH, Yang Z, Jean YH, et al. Intra-articular injection of collagenase induced experimental osteoarthritis of the lumbar facet joint in rats. Eur Spine J. 2008 May;17(5):734-42. 